\title{
APPARATUS NEW INNOVATION IN PULSED ELECTROMAGNETIC THERAPY
}

The latest innovation in the rapidly advancing world of pulsed electro-magnetic therapy comes from one of the leading companies in this field; Electro-Medical Supplies (Greenham) Limited of Wantage.

The MEGAPULSE therapy unit has been developed to give a very wide range of pulse widths and repeat rates, thereby enabling the therapist to employ a wider choice of treatments, within the known requirements, for a variety of conditions.

It will also be invaluable for the continuing research and clinical trials in this area of treatment.

The use of short-wave diathermy as a therapeutic technique was first used before 1930 but until quite recently it was generally believed that its sole effect was to increase blood flow in the zone under treatment.

However, recent world wide research has indicated that by using pulses of electro-magnetic energy under strictly controlled conditions the natural healing processes in living tissue are accelerated.

Further investigation into the effects of pulsed electromagnetism shows that it is beneficial over both areas of low sensitivity such as skin ulcers, poorly vascularised areas and a reas containing osteosynthesis and also areas having good vascula rity.

Although the process which obtains such results is not yet clear, the effect appears to be the re-polarisation of cell membranes and increased cell permeability.

It is against the background of the advances in recent years that the MEGAPULSE pulsed therapy system has been launched, bringing increased sophistication to this method of treatment.

The MEGAPULSE Hi-Q patient applicator has been designed with an internal-tuned circuit supplied with power from the radio frequency generator. When used over the a rea of treatment it produces a strong radio frequency electromagnetic field, but without giving the high powers often used previously.

The wider range of pulse widths and repeat rates that the MEGAPULSE allows can be further controlled by selection of either a normal continuous train of pulses or groups of pulses. These groups can further be controlled to deliver pulses for one third of the total treatment time (with two thirds rest period) or exactly vice versa.

A combination of continued application of the wider pulsed widths, 200 and 400 micro seconds in duration, and faster pulse repeat rates of 200 and 400 pulses per second can produce an average power from the applicator to give a thermal sensation over the treatment area in some patients.

Applying pulses in groups reduces the thermal effect so that the treatment can be assessed without complication from its by-product.

The MEGAPULSE therapy unit is a compact, easily operated unit, which will introduce a new dimension to this field of patient care.

For further information please contact the S.A. Sole Agents, Medical Distributors (Pty) Ltd, PO Box 3378. Johannesburg 2000. PO Box 195 Cape Town 8000 and PO Box 5298 Durban 4000. 